\title{
Estudio comparativo de flujos y resistencias de catéteres para hemodiálisis usando bioconectores luer-lock
}

\author{
Premio Covidien Quinton de Investigación sobre Catéteres Permanentes
}

José Luis Cobo Sánchez - $M^{a}$ Elena Incera Setién - $M^{a}$ Yolanda Vicente Jiménez - Rosana Sainz Alonso Araceli Sáenz de Buruaga Perea - Modesto Díez Rumayor - Raquel Menezo Viadero - Magdalena Gándara Revuelta - Ma ${ }^{a}$ Eugenia Cuadrado Mantecón - Camino Villa Llamazares - Rosa Alonso Nates

Servicio de Nefrología. Hemodiálisis. Hospital Universitario Marqués de Valdecilla. Santander.

\section{Resumen}

El objetivo de este trabajo es evaluar los flujos y resistencias al usar dos tipos de bioconectores luer-lock para catéteres de hemodiálisis. Como objetivos secundarios se pretende evaluar los cambios en la coagulación del sistema, el tiempo empleado en la conexión/desconexión y la opinión de enfermería respecto a su utilización.

Material y métodos: estudio experimental aleatorizado en 10 pacientes en hemodiálisis, 5 con bioconector Tego® (grupo A) y 5 con BD QSyte ${ }^{\circledR}$ (grupo B), a lo largo de 12 sesiones consecutivas ( 6 con bioconectores y 6 con conexión directa al monitor). Se estudian las siguientes variables: sociodemográficas, tipo de técnica de hemodiálisis, flujos de sangre, presión venosa, $\mathrm{Kt}$, KtV, coagulación del sistema, tiempo empleado en la conexión/desconexión y la opinión del personal de enfermería.
Correspondencia:

José Luis Cobo Sánchez

Servicio de Nefrología. Hemodiálisis

Hospital Universitario Marqués de Valdecilla

Avda. Valdecilla s/n

39008 Santander

jlcobosanchez@gmail.com
Resultados: La muestra se compuso de 6 mujeres y 4 hombres, con una edad media 62 años y un tiempo medio en hemodiálisis de 4,2 años. En el grupo A, no existieron diferencias estadísticamente significativas entre los flujos de sangre, las presiones venosas y las dosis de diálisis. El grupo B presentó diferencias estadísticamente significativas en los flujos de sangre y en las presiones venosas. No hubo diferencias entre el uso de bioconectores y la conexión directa al catéter en la coagulación del sistema. Los tiempos de conexión y desconexión disminuyeron en ambos grupos. El personal de enfermería opinó que la comodidad en el uso de bioconectores era: mucha $(82 \%)$, bastante $(9 \%)$ e igual que sin ellos $(9 \%)$.

Conclusiones: Las tiempos de conexión y desconexión disminuyen con la utilización de bioconectores. El bioconector Tego®, ofrece mejores flujos sanguíneos y menores resistencias venosas.

\section{PALABRAS CLAVE:}

- HEMODIALISIS

- CATETER VENOSO CENTRAL

- BIOCONECTOR LUER-LOCK

- DOSIS DE DIÁLISIS

- FLUJO DE SANGRE

- ENFERMERIA 


\section{Comparative study of flows and resistances of haemodialysis catheters using luer lock bioconnectors}

\begin{abstract}
The aim of this study is to evaluate flows and resistances when using two types of luer lock bioconnectors for haemodialysis catheters. As secondary objectives, the study seeks to evaluate the changes in the coagulation of the system, connection/disconnection time and the opinion of nursing staff on their use.
\end{abstract}

Material and methods: randomized experimental study of 10 patients undergoing haemodialysis, 5 with Tego ${ }^{\circledR}$ bioconnectors (group A) and 5 with BD Q-Syte ${ }^{\circledR}$ (group B), over 12 consecutive sessions ( 6 with bioconnectors and 6 directly connected to the monitor). The following variables were studied: sociodemographic, type of haemodialysis technique, blood flows, venous blood pressure, $\mathrm{Kt}$, $\mathrm{KtV}$, coagulation of the system, time used in connecting/disconnecting and the opinion of nursing staff.

Results: The sample comprised 6 women and 4 men, with an average age of 62 and an average time on haemodialysis of 4.2 years. In group $A$, there were no statistically significant differences between blood flows, venous blood pressure and dialysis dose. Group B showed statistically significant differences in blood flows and venous blood pressure. There were no differences between the use of bioconnectors and direct connection to the catheter in the coagulation of the system. Connection and disconnection times were reduced in both groups. Nursing staff considered that the convenience of the use of bioconnectors was: very convenient $(82 \%)$, quite convenient $(9 \%)$ the same as without them $(9 \%)$.

Conclusions: Connection and disconnection times fall with the use of bioconnector. The Tego $\AA$ bioconnector offers better blood flows and lower venous resistance.

\section{KEY WORDS:}

- HAEMODIALYSIS

- CENTRAL VENOUS CATHETER

- LUER LOCK BIOCONNECTOR

- DIALYSIS DOSE

- BLOOD FLOW

- NURSING

\section{Introducción}

El uso de catéteres venosos centrales (CVC) como acceso vascular para hemodiálisis (HD) está aumentando de forma exponencial en los últimos años. Según el estudio DOPPS, el número de pacientes con un CVC se ha duplicado en los últimos 10 años, tanto en Europa como en América; en nuestro país su uso se ha triplicado, pasando de un $7 \%$ en 1996 a un $21 \%$ en $2007^{1}$. Este aumento está relacionado con ciertas características del paciente actual en hemodiálisis: mayor edad, progresión más rápida de la enfermedad renal, elevada co-morbilidad cardiovascular y menores niveles de albúmina sérica ${ }^{2}$. Ser portador de un CVC conlleva un mayor riesgo de mortalidad frente a los que son portadores de una fístula, con un riesgo relativo de los portadores de catéter de 1,79 frente a 1,29 de los portadores de físitula 2 . Este aumento del riesgo, además de venir dado por el perfil citado, está relacionado con un mayor riesgo de bacteriemia derivado de su manipulación ${ }^{2-5}$. Para disminuir el riesgo derivado de la manipulación, además de un manejo adecuado de los catéteres, basados en rigurosas medidas de asepsia, existen distintos productos en el mercado como soluciones de cebado con antibiótico $0^{6,7}$ o bioconectores tipo luer-lock ${ }^{8}$.

El objetivo de los bioconectores es crear una luz sin obstáculos en la posición abierta, con una resistencia equivalente a la obtenida cuando se conecta la línea directamente al catéter. El bloqueo del conector esta formado por una capa de silicona para evitar la hemólisis. Su eficacia a la hora de disminuir o eliminar el riesgo de infección asociada a la manipulación del catéter esta demostrada-10, sin embargo existen pocos estudios que evalúen los flujos y resistencias durante la HD, ninguno en nuestro país ${ }^{11-12}$. Por ello, nuestro objetivo principal fue evaluar los flujos y resistencias 
y por lo tanto la dosis de diálisis, al usar dos tipos de bioconectores luer-lock $\left(\right.$ Tego $^{\circledR}$ y BD Q-Syte ${ }^{\circledR}$ ). Como objetivos secundarios nos planteamos evaluar cambios en la coagulación del sistema, tiempo empleado en la conexión y desconexión, así como la opinión del personal de enfermería respecto a su utilización.

\section{Material y Métodos}

Se realizó un estudio experimental aleatorizado en 10 pacientes en HD, 5 con el bioconector Tego ${ }^{\circledR}$ (grupo A) y 5 con el bioconector BD Q-Syte ${ }^{\circledR}$ (grupo B), a lo largo de 12 sesiones de HD consecutivas. Los criterios de inclusión fueron: ser paciente en HD crónica durante más de 3 meses, ser portador de un CVC tipo Tessio y aceptar su inclusión en el estudio, obteniéndose el consentimiento informado.

La aleatorización se realizó al azar, escogiendo 3 pacientes en HD convencional y 2 pacientes en HD en alto flujo por grupo. Los pacientes en HD convencional se dializaron en un monitor Integra ${ }^{\circledR}$ y los pacientes en $\mathrm{HD}$ en alto flujo en un monitor $4008 \mathrm{~S}^{\circledR}$.

Para evaluar la diferencia de flujos, resistencias y la dosis de diálisis se recogieron los flujos de sangre, la presión venosa, el Kt calculado por los monitores y el KtV calculado según la fórmula de Daugirdas, durante 6 sesiones sin usar los bioconectores y durante otras 6 sesiones usando los bioconectores. Para no alterar ninguna otra condición extrínseca, se mantuvo la dosis de heparinización y la solución de cebado del catéter durante los 30 días del estudio.

Para evaluar los cambios en la coagulación del sistema, el tiempo empleado en la conexión y desconexión, y la opinión del personal de enfermería se elaboró una hoja de recogida de datos que cumplimentaba la enfermera responsable del paciente en cada sesión. Todo el personal de enfermería fue informado por escrito de las condiciones en que se desarrollaría el estudio. Se mantuvo así mismo durante las 12 sesiones el protocolo de conexión: eliminación de 5 $\mathrm{ml}$ para desechar la solución de cebado y salinización con $20 \mathrm{ml}$ de suero salino 0,9\% y de desconexión: salinización con $20 \mathrm{ml}$ de suero salino $0,9 \%$, y cebado con la solución según cada caso y volumen estipulado en función de la longitud de cada ramal. La desinfección de los bioconectores se realizó con clorhexidina al $2 \%$, siguiendo las instrucciones de los fabricantes; se cambiaban el primer día de la semana. También se recogieron datos clínicos y sociodemográficos de la historia clínica de los pacientes.

El análisis estadístico se realizó con el paquete estadístico SPSS 15.0, utilizando para el contraste de hipótesis la prueba de los rangos con signo de Wilcoxon, el test $U$ de Man-Whitney y la prueba de McNemar Kappa. Los resultados se consideraron significativos si el nivel crítico observado era inferior al $5 \%(p<0,05)$.

\section{Resultados}

De los 21 pacientes que cumplían los criterios de inclusión, se seleccionaron 10 pacientes: 6 mujeres y 4 hombres, con una edad media de 62 años (DE: 12 años) , 4,2 años (DE: 5,1 años) en tratamiento sustitutivo renal con $\mathrm{HD}$ de media y con un tiempo medio de implantación del catéter de 21,6 meses (DE: 22,8 meses). Las características de cada paciente además de los flujos de sangre, las presiones venosas y las dosis de diálisis de ambos grupos durante el periodo de estudio se recogen en la tabla 1.

En el grupo A, no existieron diferencias estadísticamente significativas entre los flujos sanguíneos ( $p=$ $0,68)$, las presiones venosas $(p=0,22)$ y las dosis de diálisis ( $K t \mathrm{p}=0,68 ; \mathrm{KtV} p=0,62$ ), entre el uso del bioconector y la conexión directa. En el grupo B, no hubo diferencias estadísticamente significativas en cuanto a la dosis de diálisis ( $K t \mathrm{p}=0,5 ; \mathrm{KtV} p=0,68$ ); pero si existieron diferencias estadísticamente significativas tanto en los flujos sanguíneos $(p=0,043)$ como en las presiones venosas $(p=0,043)$ con el uso del bioconector. Estas diferencias estaban relacionadas con la técnica $(p=0,058)$ y el género $(p=0,048)$, presentando mejores flujos las mujeres y aquellos pacientes en hemodiálisis convencional.

En cuanto a la coagulación del sistema, no hubo diferencias entre el uso de bioconectores y la conexión directa al paciente; sólo una paciente del grupo B presentó un coágulo de sangre en el ramal venoso a la conexión, cuya extracción fue dificultosa. Los tiempos de conexión disminuyeron con el uso de ambos bioconectores en unos 2,3 minutos de media ( $p=$ 0,032 ), aunque el día del cambio del bioconector es- 


\begin{tabular}{|c|c|c|c|c|c|c|c|c|c|c|c|}
\hline \multicolumn{12}{|c|}{ Grupo A } \\
\hline \multirow[b]{2}{*}{ Paciente } & \multirow[b]{2}{*}{ Sexo/Edad } & \multirow[b]{2}{*}{ Nefropatía } & \multirow{2}{*}{$\begin{array}{c}\text { Técnica } \\
\text { HD }\end{array}$} & \multicolumn{4}{|c|}{ Sin bioconector } & \multicolumn{4}{|c|}{ Con bioconector } \\
\hline & & & & $\begin{array}{l}\text { Flujo } \\
\text { sangre }\end{array}$ & $\begin{array}{l}\text { Presión } \\
\text { venosa }\end{array}$ & Kt & KtV & $\begin{array}{l}\text { Flujo } \\
\text { sangre }\end{array}$ & $\begin{array}{l}\text { Presión } \\
\text { venosa }\end{array}$ & Kt & KtV \\
\hline Pacl & Hombre/63 & Poliquistosis & HDF & 250 & 180 & 44,8 & 1,66 & 283,3 & 153,3 & 42,5 & 1,38 \\
\hline Pac2 & Mujer /40 & N. diabética & $\mathrm{HDC}$ & 341,6 & 183,3 & 30,7 & 1,84 & 320 & 175,83 & 33,27 & 1,4 \\
\hline Pac3 & Mujer/ 55 & N. diabética & HDF & 323,3 & 193,3 & 50,5 & 1,41 & 316,67 & 180 & 47,45 & 1,47 \\
\hline Pac4 & Hombre/63 & Glomerulonefritis & $H D c$ & 310 & 190,8 & 39,4 & 1,3 & 311,67 & 204,17 & 40,53 & 0,96 \\
\hline Pac5 & Mujer/70 & Mieloma & $\mathrm{HDC}$ & 313,3 & 140,8 & 25,5 & 1,48 & 320 & 137,5 & 32,2 & 1,72 \\
\hline \multicolumn{12}{|c|}{ Grupo B } \\
\hline Pac6 & Mujer/ 59 & N. Túbulo-interst. & $\mathrm{HDC}$ & 300 & 183,3 & 34,2 & 1,65 & 286,67 & 191,67 & 32,78 & 1,66 \\
\hline Pac7 & Hombre/65 & Nefroangioescler. & HDF & 266,6 & 174,1 & 38,5 & 1,36 & 260 & 190,83 & 36,92 & 1,25 \\
\hline Pac8 & Mujer/65 & Nefroangioescler. & $\mathrm{HDC}$ & 293,3 & 203,3 & 24,8 & 0,87 & 283,3 & 222,5 & 31,15 & 1,12 \\
\hline Pac9 & Hombre/56 & Desconocida & HDF & 262,5 & 171,6 & 39,4 & 1,11 & 250,83 & 173,3 & 37,48 & 0,97 \\
\hline Pac10 & Mujer/89 & Nefroangioescler. & $\mathrm{HDC}$ & 286,6 & 170,8 & 34,1 & 1,55 & 273,3 & 187,5 & 28,83 & 1,32 \\
\hline
\end{tabular}

Tabla 1.- Características de los pacientes estudiados, flujos de sangre en $\mathrm{ml} / \mathrm{min}$, presiones venosas en $\mathrm{mmHg}$ y dosis de diálisis en litros; según grupos y tiempos de medición. HDc: hemodiálisis convencional; HDF: hemodiálisis de alto flujo.

tos tiempos son muy similares a la conexión directa. La reducción de los tiempos de desconexión en ambos grupos fue más considerable, unos 4,5 minutos menos $(p=0,01)$. La opinión de las enfermeras en el uso de bioconectores fue muy cómodo $(82 \%)$, bastante cómodo ( $9 \%$ ) e igual que sin ellos $(9 \%)$.

\section{Discusión}

Según nuestros resultados, parece que el bioconector del grupo B disminuye el flujo sanguíneo y aumenta la presión venosa con respecto a la conexión directa de las líneas al catéter. Eloot et al estudiaron in vitro ${ }^{11}$ los flujos y resistencias de 3 tipos de bioconectores para catéteres de HD $\left(\operatorname{Tego}_{0}{ }^{\circledR}, \mathrm{BD}-\mathrm{Q}\right.$ Syte $^{\circledR}$ y $\operatorname{Codan}^{\circledR}{ }^{\circledR}$, utilizando agua como sustituto Newtoniano de la sangre, usando distintos flujos de bomba y en distintos monitores. Encontraron diferencias significativas tanto en los flujos arteriales como en las presiones venosas entre ellos, encontrando resultados significativamente mejo- res con el Tego ${ }^{\circledR}$ que con los otros dos bioconectores. Achacaron estas diferencias a que el botón de silicona del BD Q-Syte ${ }^{\circledR}$ se encuentra posicionado en la parte externa del mismo, por lo que la posición y el sistema de apertura son distintos a los otros 2 bioconectores. Indicaban también que las líneas influían en la apertura de los distintos dispositivos. En nuestra muestra, los cambios en los flujos y resistencias en el grupo $B$ estaban relacionados con la técnica de $H D$, por lo que las líneas también son distintas, pudiendo ser una de las causas por las que varían los flujos y resistencias. Un estudio reciente de este mismo grupo ${ }^{13}$, en el que además de incluir resultados in vitro, estudiaron en 11 pacientes ( 8 con catéteres de una sola luz y 3 con doble luz) los flujos y resistencias con los mismos bioconectores, observaron roturas del dializador con bioconectores Tego ${ }^{\circledR}$ y $\operatorname{Codan}^{\circledR}$, pero ninguna con BD QSyte ${ }^{\circledR}$; por lo que concluyeron que este último sería el más adecuado y seguro de los 3 dispositivos. En nuestro estudio no encontramos ni roturas de dializador ni mayores problemas de coagulación. 
Franco et $\mathrm{al}^{12}$ estudiaron los flujos y resistencias en 23 pacientes de 3 a 9 sesiones de HD, con distintos tipos de catéteres y monitores, usando el bioconector $\mathrm{Tego}^{\circledR}$. Sus flujos sanguíneos oscilaron entre 281 y $330 \mathrm{ml} / \mathrm{min}$ con unas presiones venosas entre 103 y $219 \mathrm{mmHg}$. Los flujos y resistencias en nuestro estudio con el mismo bioconector fueron mayores, aunque puede ser debido a que hubo una mayor uniformidad en la muestra, ya que como se ha comentado, parece que los distintos tipos de líneas influyen de forma considerable. Este estudio ${ }^{12}$ no compara los resultados de Tego ${ }^{\circledR}$ con los flujos y presiones venosas en esos mismos pacientes sin usar el bioconector, pero si incluyen la resistencia arterial que ofrece el catéter (de -163 a $-224 \mathrm{mmHg}$ ); dato que no hemos registrado en nuestro estudio y que podría ser interesante registrar en próximas investigaciones.

No hemos encontrado ningún estudio que evaluase ni los tiempos de conexión y desconexión ni la comodidad de las enfermeras en el uso de los bioconectores, aunque a la vista de los resultados, parece que disminuyen estos tiempos y facilitan la labor de enfermería en ambos casos.

\section{Conclusiones}

Existen diferencias a favor del bioconector Tego ${ }^{\circledR}$ que ofrece mejores flujos sanguíneos y menores resistencias venosas que el BD Q-Syte ${ }^{\circledR}$, en consonancia con los estudios in vitro, disminuyendo los tiempos de conexión y desconexión. Sin embargo, deben realizarse más estudios comparativos, experimentales y aleatorizados para llegar a conclusiones que puedan generalizarse.

\section{Bibliografía}

1. Ethier J, Mendelssohn DC, Elder SJ, Hasegawa T, Akizawa T, Akiba T, Canaud BJ, Pisoni RL. Vascular access use and outcomes: an international perspective from the Dialysis Outcomes and Practice Patterns Study. Nephrol Dial Transplant 2008; 23 (10): 3219-3226.

2. Pisoni RL, Arrington CJ, Albert JM, Ethier J, Kimata N, Krishnan M, Rayner HC, Saito A, Sands JJ, Saran R, Gillespie B, Wolfe RA, Port FK. Facil- ity hemodialysis vascular access use and mortality in countries participating in DOPPS: an instrumental variable analysis. Am J Kidney Dis 2009; 53(3):475-491.

3. Lemaire $X$, Morena $M$, Leray-Moragués $H$, Henriet-Viprey D, Chenine L, Defez-Fougeron C, Canaud B. Analysis of Risk Factors for Catheter-Related Bacteremia in 2000 Permanent Dual Catheters for Hemodialysis. Blood Purif 2009; 28(1):21-28.

4. Jean G, Charra B, Chazot C, Vanel T, Terrat JC, Hurot JM, Laurent G. Risk factor analysis for longterm tunneled dialysis catheter-related bacteremias. Nephron 2002; 91(3):399-405.

5. Marr KA, Sexton DJ, Conlon PJ et al. Catheter-related bacteremia and outcome of attempted catheter salvage in patients undergoing hemodialysis. Ann Intern Med 1997; 127: 275-280.

6. Elcano Cuesta I, Usoz Guembe A, Moriones Lopetegui L, Loperena Eslava C, Zoco Goñi M, Arregui $E$ et al. Carta al director: detección precoz de la colonización de catéteres venosos tunelizados en pacientes en una unidad de hemodiálisis. Eficacia del sellado con antibiótico para erradicar dicha colonización. Rev Soc Esp Enferm Nefrol 2005; 8(1):77-78.

7. Baltrons Bosch A, Coll Vinyoles S, Font Senen C, Jubany López M, Plana Illa C, Sunyer García A. Estudio comparativo del sellado de catéteres con citrato trisódico o heparina sódica más gentamicina. Rev Soc Esp Enferm Nefrol 2008; 11 (2): 95-100.

8. McAfee N, Seidel K, Watkins S, Flynn J. Use of Tego Connectors to Prevent Hemodialysis Catheter Infections in Children. Am J Kidney Dis 2009; 53(4):B37.

9. Bouza E, Munoz P, Lopez-Rodriguez J et al. A needleless closed system device (CLAVE) protects from intravascular catheter tip and hub colonization: a prospective randomized study. J Hosp Infect 2003; 54: 279-287.

10. Casey $A L$, Worthington $T$, Lambert PA et al. A randomized, prospective clinical trial to assess the 
potential infection risk associated with the PosiFlow ${ }^{\circledR}$ needleless connector. J Hosp Infect 2003; 54: 288-293.

11. Eloot S, De Vos JY, Hombrouckx R, Verdonck P. How much is catheter flow influenced by the use of closed luer lock access devices? Nephrol Dial Transplant. 2007; 22(10):3061-4.
12. Galli F, Masciullo M, Gibilisco P. Effective Central Venous Catheter Hemodialysis with a Novel Needlefree Connection Device. S. Maugeri University Hospital, Hemodialysis Unit, Pavia, Italy. Artículo en internet. Disponible en: http://www.setfinder. com/Docs-Tego/M1-1153-Tego-Hemodialysis.pdf

13. De Vos JY, Hombrouckx R, Eloot $S$. How much is catheter flow influenced by use of closed luer lock access devices? $37^{\text {th }}$ EDTNA/ERCA International Conference Abstract Book: 13-14. Prague; 6-9 September 2008. Paris: European Dialysis and Transplant Nurses Association/ European renal Care Association. 